\title{
The Football of Logic
}

\author{
Fabien Schang \\ Universidade de Estadual de Maringá, \\ Brazil
}

e-mail: schangfabien@gmail.com

\begin{abstract}
An analogy is made between two rather different domains, namely: logic, and football (or soccer). Starting from a comparative table between the two activities, an alternative explanation of logic is given in terms of players, ball, goal, and the like. Our main thesis is that, just as the task of logic is preserving truth from premises to the conclusion, footballers strive to keep the ball as far as possible until the opposite goal. Assuming this analogy may help think about logic in the same way as in dialogical logic, but it should also present truth-values in an alternative sense of speech-acts occurring in a dialogue. The relativity of truth-values is focused by this way, thereby leading to an additional way of logical pluralism.

Keywords: assertion, denial, football, game, goal, logic, possession, speechacts, truth-values, strategy, tactics.
\end{abstract}

\section{Introduction: Logic of Football vs Football of Logic}

Football (or the American "soccer") is not a serious thing, like logic. Or it should not be so, for those who see nothing in it but a distraction for the masses. Now such a game may be taken seriously, even if its usual depiction in terms of star salaries and hooliganism reduces this sport to a desperate feature of entertainment society. Game-theorists do know that games may be taken seriously. As a matter of fact, it can be said about football what the social constructivist Robert Cox claimed about states in the area of international relations, i.e., that they are what is done with them. What is or can be done with football, from a serious point of view? Our answer is: an explanatory model of logic, from a game-theoretical perspective. There are at least two reasons not to proceed in this way. On the one hand, such an approach to logic already exists under the heading of Lorenzen and Lorenz's dialogical logic [4], or Hintikka's Game-Theoretical Semantics [3]. So why bother with a provocative introduction of football in the very serious area of logic? On the other hand, philosophers who are reluctant to chiasms should note that there may be a "logic of football" in the sense of a set of recursive rules explaining how football proceeds, but not the converse. No serious sense should be given to an alleged "football of logic", accordingly, if such an expression assigns an explanatory role of football to logic but not the contrary one. And yet, the next sections are meant to show to what extent the game of football may throw some light on the game of logic. Or, at the least, that they can be compared with each other with no prominent role for either. A first 
logical reflection in football has been already made recently, in a paper where the activity of refereeing was compared to a peculiar game whose rules differ from the players' ones [5]. The present paper purports to push the logical line farther, accounting for inference rules of logic by means of the rules surrounding the football players themselves.

\section{Question-Answer Game}

The sort of game we want to focus now is a question-answer game of logic. The explanation borrows from a four-valued logic of acceptance and rejection $\mathbf{A} \mathbf{R}_{4}$ [11]. Roughly speaking, it is a logic of information including a set of formulas and logical constants (negation, conjunction, disjunction, and a strong implication) interpreted into a domain of four structured values with Boolean elements. The reason why there are four logical values comes from the combination of single Boolean values depicted in the tradition of Belnap and Dunn's logic FDE [1]: replacing truth and falsity by data (or evidence) for or against a given proposition, it becomes possible to have inconsistent data where a statement is said to be both true or false. In the contrary case, it is said to be neither true nor false where no information is available. By doing so, this logical system endorses rejectivism - the linguistic or logical theory according to which negation is primarily a speech-act to be explained in terms of denial. By explaining the background of rejectivism and depicting the no-answer as a force indicator that plays the role of denial [12], it results in another view of negation as an opposite of affirmation. Thus, the two polar answers "yes" and "no" are the basic units of meaning conveyed by affording some information with a sentential content. There is no eternal Proposition descending from the Fregean "Third Realm" of thoughts [7], in such a perspective; that is, truth and falsity are nothing but commitments made by a speaker about what is accepted or strongly rejected in the speaker's belief set. There is no sufficient space to discuss at length about the epistemological stakes of rejectivism, in the following paper [10]. Instead, let us focus on the technical peculiarities of the four-valued system $\mathbf{A} \mathbf{R}_{4}$.

One feature is the structured form of valuations $\mathbf{A}(p)=\left\langle\mathrm{a}_{1}(p), \mathrm{a}_{2}(p)\right\rangle$ in $\mathbf{A} \mathbf{R}_{4}$, which consists in an ordered pair of answers $\mathrm{a}_{1}(p)$ and $\mathrm{a}_{2}(p)$ to whether there is evidence for or against a given sentence $p$, respectively. There are only two possible answers: yes (1), or no (0), A being a function mapping each answer $\mathbf{a}_{i}(p)$ onto 1 or 0 . For sake of simplicity, the logical values of $\mathbf{A} \mathbf{R}_{4}$ will be simplified in the form $(x, y)$, where $x=\mathbf{a}_{1}(p)$ and $y=\mathbf{a}_{2}(p)$.

Another feature is the ensuing valuation function $\mathbf{A}(p)$, which applies from a given sentential content $p$ to the set of logical values $\mathbf{4}=\{11,10,01,00\}$. The common point with FDE is the number of values and their informal interpretation: 11 means "true and false" ("both true and false" or B, in FDE), 10 means "true and not false" ("true only" or T, in FDE), 01 means "not true and false" ("false only" or F, in FDE), and 00 means "neither true nor false" ("None" or N, in FDE).

\section{Strong Conditional}

What of the core issue of this special volume: conditional, i.e. implication? The main advantage of the above valuation is to afford a stronger characterization of it; stronger, in the sense that some of the theorems where the material implication of classical logic essentially occurs do not hold in $\mathbf{A} \mathbf{R}_{4}$. Our logical constant is a case of "defective" conditional [14], which means that the logical relation cannot be satisfied unless the antecedent is said true or, equivalently, accepted by the speaker. Such a feature cancels any form of the highly counterintuitive paradoxes of material implication, by virtue of which the whole relation holds once the antecedent is false. A proper definition of any logical constant amounts to specify the conditions under which the compound sentence can be told true or told false, given that these two truth-values are independent from each other in our rejectivist approach. Negation, conjunction and disjunction are defined in $\mathbf{A} \mathbf{R}_{4}$ in the same way as in FDE. Conditional is defined in a non-standard way, however. 
On the one hand, any conditional $p \rightarrow q$ is true, i.e. accepted by the speaker, if and only if both $p$ and $q$ are accepted. In symbols:

$\mathbf{a}_{1}(p \rightarrow q)=1$ iff $\mathbf{a}_{1}(p)=\mathbf{a}_{1}(q)=1 ; \mathbf{a}_{1}(p \rightarrow q)=0$, otherwise

On the other hand, $p \rightarrow q$ is rejected, i.e. strongly denied by the speaker, if and only if $p$ is accepted and $q$ is rejected. In symbols:

$$
\mathbf{a}_{2}(p \rightarrow q)=1 \text { iff } \mathbf{a}_{1}(p)=1 \text { and } \mathbf{a}_{2}(q)=1 ; \mathbf{a}_{2}(p \rightarrow q)=0 \text {, otherwise }
$$

Note that any no-answer does not mean a rejection but, rather, a mere denial of the speaker (for want of any sufficient evidence for or against the corresponding sentence). This means symbolically that $\mathbf{a}_{1}(p)=0$ need not entail that $\mathbf{a}_{2}(p)=1$. Note also that only the truth-condition of strong conditional $\rightarrow$ differs from that of classical or material implication $\supset$, which assumes that not affirming and rejecting are on a par:

$$
\mathbf{a}_{1}(p \supset q)=1 \text { iff } \mathbf{a}_{2}(p)=1 \text { or } \mathbf{a}_{1}(q)=1 ; \mathbf{a}_{1}(p \supset q)=0 \text {, i.e. } \mathbf{a}_{2}(p \supset q)=1 \text {, otherwise. }
$$

A number of classical theorems about implication are not valid any more in $\mathbf{A} \mathbf{R}_{4}$, symbolized by the non-truth-preserving relation $F^{*}$ :

(1) $p \rightarrow q$ F $^{*} \neg p \vee q$

(2) $p \rightarrow q k^{*} \neg q \rightarrow \neg p$

(3) $k^{*} p \rightarrow(q \rightarrow p)$

(4) $\mathrm{F}^{*}(p \rightarrow q) \rightarrow((q \rightarrow r) \rightarrow(p \rightarrow r))$

The failure of (1) is due to the failure of the paradoxes of material implication. The failure of (2) concerns the classical formulation of Modus Tollens (MT) and relates to the distinction between sentential negation and rejection in $\mathbf{A} \mathbf{R}_{4}$. The failures of (3) and (4) rely on the defective import of strong conditional, to the effect that no conditional holds once its antecedent is not accepted.

At the same time, some fundamental properties of implication are preserved with strong conditional, with respect to the general relation of logical consequence:

(5) $k p \rightarrow q$ iff $p$ F $q$

(6) $p \rightarrow q, p \neq q$

(7) $p \rightarrow q, q \rightarrow r \vDash p \rightarrow r$

(5) is the Deduction Theorem, which means that strong conditional preserves truth once the antecedent is accepted; (6) is an expression of Modus Ponens (MP), just as (7) by contrast to the failure of the object language version of transitivity in (4). The main advantage of strong conditional is to avoid the paradoxes of material implication while avoiding any collapse with conjunction. Importantly, a central feature of conditional is lost in its lattice theoretical definition:

$$
v(p \rightarrow q)=1 \text { iff } v(p) \leq v(q)
$$


While this feature is mostly maintained as an essential property of implication in the literature (see Costa-Leite's paper, in the present issue), we consider the paradoxical behavior of material or classical implication as a by-product of it. At the same time, the failure of (MT) in (2) is taken to be a much more important result of $\mathbf{A} \mathbf{R}_{4}$. If (MP) and (MT) are viewed as essential properties of implication, strong conditional must be adjusted to (MT) in order to be considered as a proper characterization of conditionality. This is done by redefining "Tollens" as a non-falsity preserving relation from $q$ to $p$, rather than a truth-preserving relation from $\neg q$ to $\neg p$ [9]. In a nutshell, (MT) means the following: let a given speaker accept a conditional relation from $p$ to $q$; if this speaker also assumes that the consequent $q$ is not true in a given context, then it is not possible any more to make the whole conditional true in such a context, due to the defective import of our strong conditional; the least thing to do in order not to lose the game is to deny the antecedent, for such a conditional is made false exactly when $p$ is true and $q$ is false. This means that a player may not win without losing, either. The classical characterization of conditional results in a "all or nothing" or bivalent situation, and that is the reason why (MT) is depicted as it stands in classical logic. In our case, however, the gap between winning and losing a game is filled by weak denial, which corresponds to a case of draw. You can compare this case with dialogical logic, when the attacked player avoids asserting something in order not to make a fatal move and wants to extend the duration of the game as far as possible. The moral of this is that $\mathbf{A} \mathbf{R}_{4}$ endorses a twofold view of inference: as a maximal, truth-preserving relation, on the one hand; as a minimal, non-falsity preserving relation, on the other hand. This echoes with some other words around many-valued inference.

We just described the meaning of strong conditional. Now let us consider its meaning from a special game-theoretical point of view that of football, together with the other logical constants of negation, conjunction, and disjunction.

\section{Analogical Games}

Comparison is not reason, admittedly. But comparison is clarification, and the present table proposes a couple of analogies between the lexical fields of football and logic. By the same way, it helps make sense of a pluralist view of logic including more than only one game strategy. Assuming a Tarskian view of logical as a theory of truth-preserving relation, let us see how rejectivism, negation and strong conditional may make sense in such a perspective.

\begin{tabular}{|c|c|}
\hline Football & Logic \\
\hline having the ball & truth \\
\hline losing the ball & falsity \\
\hline game fact & (provisory) conclusion \\
\hline match (finite set of actions) & reasoning (finite set of inferences) \\
\hline goal (successful shot) & valid inference \\
\hline failed shot & invalid inference \\
\hline own-goal & counter-inference \\
\hline coach & reasoner \\
\hline players & atomic sentences \\
\hline attack & assertion \\
\hline defense & rejection \\
\hline ball & interpretation function \\
\hline ball preservation & logical consequence \\
\hline referee & logic \\
\hline
\end{tabular}


Let us now translate the logical background into our paradigm of football. As depicted by the above table, football is a game in which a team strives to keep the ball in order to score a goal at least once more than the other team. The usual opposition between a Proponent and its Opponent in dialogical logic is taken for granted, hereby: the Proponent is any team performing a finite number of moves towards scoring a goal, while it is taken for granted that the Opponent is the opposite team within a kind of zero-sum game - whoever wins makes the other lose, although draw happens once both sides are not able to fulfill their requirement. The duration of a match is finite, and a number of tactics are displayed between the players of a same team in order to fulfill the expected task into a limited amount of time.

The values of truth and falsity are context-dependent and relative to the team possessing the ball; the latter plays the role of the function interpretation $\mathbf{A}(p)$, assigning truth with respect to the attacking team and falsity with respect to the defending team. It means that truth-values are like game situations, when the team having the ball tries to go forward until the opposite goal. A team has the ball and goes forward when $\mathbf{a}_{1}(p)=1 ; \mathbf{a}_{1}(p) \neq 0$, otherwise. It does not have the ball and goes backward when $\mathbf{a}_{2}(p)=1$, and $\mathbf{a}_{2}(p) \neq 0$ otherwise. Note that this analogy may also work with American football, where players have to progress through field yards until the adversary's opposite field. In other words, truth is what is to be preserved by a given team from the beginning to the end of an articulate action; on the contrary, falsity must be avoided by any team in order not to lose before the deadline. A game is like a sequence of related sets of moves, each of these leading either to a successful or unsuccessful action.

An intended advantage of four-valuedness is the asymmetry displayed between truth and falsity, on the one hand, acceptance and rejection on the other hand. Indeed, the team that does not have the ball may not be scored as it stands and is expected to defend its own goal so long as the ball is controlled by the opposite team. For instance, the team progressing with the ball attempts to score a goal just as a Proponent wants to go from premises to the conclusion without losing truth on the way of its whole thesis; and the defenders avoid being scored by defending well or intercepting the ball anew, just as the Opponent blocks the way from true premises to true conclusion by rejecting the truth of sentences or showing the falsity of the final sentence in a given inference.

In summary, our rejectivist reading of truth-values matches with the difference in football between defense and counterattack: a team may defend without counterattacking, just as an Opponent may undermine the truth-preserving course of the Proponent without asserting the falsity of the conclusion at hand. The latter may be merely untrue, for want of any available evidence, in a material sense, or deductive relation in a formal sense of truth.

\section{Tactics}

The well-known distinction between tactics and strategy is rendered hereby by a difference between molecular sentences and inferences. We pay attention now to the former, which has to do with the definition of logical constants. The meaning of a logical constant $\bullet$ is given by a way to preserve the ball between any teammates $p$ and $q$, in our "football of logic". Progression on the field is viewed according to the ball owner. In terms of speech-acts, assertion is an offensive move performed by a speaker and expressed by a sentence, whether affirmative or negative; mere denial (no-answer to either truth or falsity) is a defensive move, whereas rejection or strong denial (yesanswer to falsity) is an attack on its own viewed from the opposite perspective as a counterattack. Logical constants can then be defined as follows. Conjunction is like a one-two pass between a pair of teammates: the ball is preserved if the move is achieved; it is lost if the second player cannot keep the ball, or if the first player cannot do what he intended with the expected receiver. Disjunction, on the other hand, makes room for an alternative range of receivers to preserve the ball and progress with it. Negation is a reversed move, thereby taking the ball again in one way of another after defending or conversely. The most intriguing constant is that of our strong conditional, defined in a defective way in $\mathbf{A} \mathbf{R}_{4}$. In our football of logic, its usual or classical truthconditions cannot be maintained because of its too weak condition for keeping the ball: it is not 
enough for any two teammates to take the ball back or having it in order to give a genuine description of a "conditional" move of the form $p \rightarrow q$. If a player $p$ has the ball, then the team is progressing forwards only if the ball is passed to the player $q$ and in no other way. Conversely, the falsity-condition of conditional corresponds to a situation in which $p$ 's team is forced to go backwards after $q$ lost the ball. The aforementioned distinction between strong and weak denial is a distinction between going backwards and being blocked by the opposite defense. If $p$ has the ball and passes to $q$ who loses it, the ball is recuperated by the other team; this is a counterpart of strong denial or rejection, i.e., $\mathbf{a}_{2}(p \rightarrow q)=1$. If $p$ has the ball and does not pass it to $q$, however, the ball may not be lost but the whole team does not progress forward; this is a counterpart of weak denial, i.e., $\mathbf{a}_{1}(p \rightarrow q)=0$. The paradox of material implication corresponds to a situation in which the tactics realized by a team is at odds with what is naturally called by an implication. For when the antecedent is not affirmed, the situation is as if the team action has been aborted, thus borrowing from Quine's account on what conditional means informally [6, p. 21]:

If the antecedent turns out to be false, our conditional affirmation is as if it had never been made.

After describing the moves made between players, we described what is meant by the logical constants of a logical system. As to the close relation between conditional and inference (or logical consequence), it corresponds to the essential difference between a means and an end: conditional is a means to go forwards by a necessary move from the player $p$ to the player $q$; inference is the general aim of going forward until the opposite goal and more often than the opposite team. Conditional is nothing but one move to do so, in addition to negation, conjunction, and disjunction. The resemblance between conditional and consequence is due to their necessary forward movement from a point to another one: from a player to another one, with conditional; from a side to the other one, with inference.

Let us now consider inference in more details, especially with respect to the way of inferring through the central constant of the present issue: conditional.

\section{Strategy}

Strategy is meant as a selection of ordered tactics. When a team is endowed with a number of various players, a good coach is the one who takes the best decision with respect to their features and the expected result of winning the game. Now just as a linear logician treats propositions like limited and decreasing resources, a coach may consider that his players are not in position to achieve some offensive moves without undergoing fatal counterattacks. If so, then the team must reinforce its defense and rely upon some mistakes from the opposite team. Although attack is taken to be the best defense, the latter remains the best way not to lose when attackers are without sufficient resources to perform their expected function. Our defective view of conditional and the resulting minimal definition of inference have to do with the previous Italian football style of "catenaccio", which consists in letting the opponents attack without intending to take the ball again and by merely blocking their attempts. A good illustration of this spoiling strategy is systematic offside. Being offside is an irregular situation that cancels an attempted instance of inference from premise to conclusion; it does not enable to win the game of truth-telling throughout, as the case turns out to be with the looser truth-conditions of classical conditional. But it does not make lose one, either: a bivalent reading would present offside as a situation leading the sanctioned team to perform something like an own-goal, assuming that any move amounts either to scoring or being scored. As for the case in which the speaker rejects the truth of the consequent, the situation is more awkward since the player does not stand "off" the game by doing so: he can lose it, in case he then affirms the truth of the antecedent. Truth counts above all, admittedly: in football, the best way not to lose is to score more goals than the opposite team in order to win the match; but also, a defensive strategy can be viewed as a complementary strategy purporting not to be scored, that is, not to lose 
the match in the end. You can compare assertions with an offensive strategy, that of scoring goals. Now an assertion can be affirmative $\left(\mathbf{a}_{1}(p)=1\right)$, or negative $\left(\mathbf{a}_{2}(p)=1\right)$ as with strong denial or rejection. In the latter case, the search for falsity-claims might appear contrary to the logical purpose of preserving truth, just as it may seem irrational for a football team to play by scoring own goals. And yet, we can even imagine such queer games in which moves that help to win in one game are moves that make one lose in another version of this game.

The plurality of strategies may be equated with the realm of non-classical inferences in logic: when more than one pattern of inference is admitted beyond the mainstream Tarskian, truthpreserving relation from premises to conclusion. The latter matching with offensive strategy, a defensive one is like a non-falsity preserving relation. In the case of conditional, again, the two main properties (MP) and (MT) may help to illustrate this point. (MP) is nothing but a correct application of the conditional relation from $p$ to $q$, given the successful tactics leading from a player $p$ to his teammate $q$. With (MT), the defensive objective is not to go forward but, after being deprived of the ball, to avoid going backward by blocking the opposite move. This is made by blocking the false transition from a true antecedent $p$ to a false consequent $q$. Given the valid inference from $p$ to $q$, and given the non-truth of $q$, what the player $p$ has to do is to not give the ball to $q$. Therefore, MT does not mean the success of an offensive strategy but, rather, the application of a least defensive strategy in order not to lose ground. Rather than winning a game, (MT) is to be viewed as a minimal rule that helps not to lose by playing logic in a reasoning including conditional.

\section{Action}

A final relevant comparison relates speech acts and actions in a game. In our approach implemented by the logical system $\mathbf{A} \mathbf{R}_{4}$, truth and falsity are not abstract properties of sentences but actions made by them (see Trafford's paper, in the present volume). Taking sentences to be players, this means that no game action can be performed without a player to pass the ball, center towards a teammate, block the opposite offensive, counterattack after taking the ball back, and the like. Moreover, we said earlier that a common action can be viewed as an offensive or defensive phase of the game, depending upon the perspective from which the action is viewed. This is another reason not to ascribe truth-values to sentences as unique and abstract entities; these values are more like contextdependent data assigned to players, with limited resources and in a limited lapse of time. For this reason, the well-known distinction made by Dummett [2] between two senses of conditional hardly seems to make sense in our game-theoretical reading of logic.

According to Searle \& Vanderveken, speech-acts are the primary vehicles of meaning; every speech-act is of the logical form F $p$, where $\mathrm{F}$ is a so-called "illocutionary force" and $p$ a sentential content. It clearly appears that affirmations and denials, i.e. yes- and no-answers, are counterparts of $\mathrm{F}$ in the metalanguage of $\mathbf{A} \mathbf{R}_{4}$. After assuming this background, an illocutionary account of conditional is proposed by Searle \& Vanderveken in the vein of Dummett's analysis [13, p. 5]:

It is essential to distinguish between a conditional speech act and a speech act whose propositional content is a conditional. In a conditional speech act expressed by a sentence of the form "If $p$ then $\mathrm{F}(q)$ " the speech act expressed by " $\mathrm{F}(q)$ " is performed on condition $p$. Syntactically the "if" clause modifies the illocutionary force indicating device. This form is quite distinct from that of the speech act performed by an utterance of a sentence of the form " $\mathrm{F}$ (if $p$ then $q$ )" whose propositional content is conditional, for in this case an illocutionary act of force $\mathrm{F}$ is categorically performed. Thus, for example, in a bet on a conditional of the form (1) "I bet you five dollars that if a presidential candidate gets a majority of the electoral votes he will win" one either wins or loses five dollars depending on the truth or falsity of the conditional proposition (provided all the presuppositions hold). On the other hand, in a conditional bet of the form (2) "If Carter is the next Democratic candidate, I bet 
you five dollars that the Republicans will win", there is a winner or a loser if Carter is the next Democratic candidate. The logical form of (2) is $p \rightarrow \mathrm{F}(q)$. This conditional is not truth-functional, for from the fact that Carter does not run for the presidency, it does not follow that every speaker performs a conditional bet of the form (2).

The whole explanation relies on the view that truth-functionality has to do with truth and falsity, together with the assumption that these truth-values result in a winning or losing bet in every case. Since the second form of conditional may lead to neither, Searle \& Vanderveken conclude from it that both conditionals do not mean the same. However, our account of conditional proposes a uniform explanation of how the speaker may win or lose the bet by putting a conditional expression. Unlike the above passage, rejection does not lead to non-truth-functionality when it means a mere no-answer. There are three truth-functionally different results for the speaker described by the above situation, in $\mathbf{A} \mathbf{R}_{4}$ : either the speaker of (2) asserts that Carter will be candidate and argues that the victory of Republicans will follow from it. In this case, the speaker is wrong and loses the bet since Carter did win the US elections in 1976. Or, he asserts the same antecedent while asserting that the Republicans will lose accordingly, in which case the speaker is right and wins the bet. Otherwise, the speaker may remain silent about whether Carter will be candidate or not; if so, then the whole conditional is merely rejected as well. This result is insightful inside our four-valued logic, however. Actually, there is nothing but a pragmatic difference between (1) and (2): in (1) the speaker commits in the truth of the antecedent, whereas in (2) (s)he does not commit in merely asserting a conditional relation between $p$ and $q$. In our football of logic, it is as if a coach generally asserts that a move from $p$ to $q$ will turn out successful for the team. Why not doing it concretely, if so? Logically speaking, the point is that the actual assertion of $p$ and $q$ following the assumedly successful relation of the conditional $p \rightarrow q$ does not seem to differ from the conjunction $p \wedge q$ : their success-conditions are the same, given that a rejection of the antecedent $p$ does not make the whole relation true any longer in $\mathbf{A} \mathbf{R}_{4}$. Nevertheless, there is no collapse of conditional to conjunction because their falsity-conditions differ: the speaker is wrong only if $q$ does not follow from $p$ once $p$ is true, whereas nothing wrong is said once the antecedent $p$ is not true.

Let us give two samples of logical inferences, the one being successful and the other unsuccessful.

The successful game fact is a valid inference is an instantiation of disjunctive syllogism, or Modus Tollendo Ponens (MTP): $p \vee q, \neg q$ F $p$.

Let us assume that the player $p$ has the ball. Then $p$ has the choice between two options in order for his own team to keep the ball: either keeping the ball for himself, or passing it to his teammate $q$. Now let us assume also that $p$ does not want to pass the ball because of $q$ being located in an inappropriate position (offside, or marked by an opponent very closely). Therefore, $p$ decides to keep the ball in order for his team not to lose it.

The unsuccessful game fact is an invalidation of the current Modus Tollendo Tollens (MTT): $p \rightarrow q, \neg q$ F $\neg p$.

Any player $p$ having the ball must pass it to his teammate $q$, if he wants his team not to lose it. Let us assume that this conditional requirement is supplemented with a circumstance in which $q$ is losing the ball. Contrary to the standard interpretation of Modus Tollendo Tollens, the conclusion hereby is by no means that the player $p$ should lose the ball either. For why on earth $p$ should lose the ball under the pretext that $q$ already lost it beforehand? A better view of our stronger reading of conditional is discussed at length in [9] and runs as follows. First of all, sentential negation and denial do not mean the same: a speaker may deny $p$ without being in position to assert its falsity, $\neg p$. From a football game perspective of logic, such a difference is on a par with that between losing the ball and not having the ball, respectively. Correspondingly, the standard reading of Modus Tollens Tollens is blamed for deriving a meaningless conclusion to the effect that the player $p$ must lose the ball if, by assuming that $p$ must pass the ball to $q$ in order for their team to keep it, $q$ 
turns out to lose it. There is merely no logical relation between these distinctive data, actually. Alternatively, our stronger reading of implication helps to redefine MTT by replacing sentential negation by denial: the first premise is to the effect that $p$ must pass the ball to $q$, in order for his team to keep the ball; now the second premise is not that $q$ lost the ball but, rather, that $q$ does merely not have the ball. For $q$ would have received it otherwise, in accordance to the conditioned way of keeping the ball by passing the ball from $p$ to $q$. From these two premises, it can thus be inferred that $p$ does not have the ball either.

To sum up, conditional is never non-truth-functional in our dialogical explanation of how it means to make a consequent conditional upon an antecedent. At the same time, Dummett is still right in saying that there is a relevant difference between betting on a conditional proposition and making a bet conditional. For the two actions do not lead to the same result, and we can express such a difference by distinguishing affirmation and denial as two genuinely informative values.

\section{Conclusion: Towards a Pluralist Football?}

The present paper has attempted to use the background of football as a game-theoretical framework for logical reasoning. A normal stance should consist in following a contrary process, i.e., making use of logic as a proper explanatory model of football rather than the converse. However, any analogy between the two activities does not impose any ordering explanatory relation between them. In other words, truth in logic is like ball possession in football and conversely; scoring is like preserving truth until the conclusion, given that the opposite goal represents a successful conclusion; the various ways for footballers of keeping or taking the ball back are like the various ways of having evidence for the truth of propositions; and the like.

Let us close this sketchy approach to a football of logic by reopening the question of logical pluralism, and its significance in the area of sports like football. What should it mean to accept more than one consequence relation in a sport game? Card games already give some such exemplifications, when the normal rules of a card game are deeply modified and can even go on inverting the usual purpose - think about cases where the worst player turns into the best one, thereby finding strategies to lose from the standard game in order to win the game from the nonstandard one. In football like in science, success is the main target in that telling the truth is as much central for a normal logician as scoring for footballers. Such alternative ways of playing logic have been illustrated elsewhere, including the case of Indian logics and their emphasis on non-standard targets like peaceful agreement with tolerance in dialogues [8]. Zero-sum games are so conspicuous that it may seem difficult to imagine any dialogical explanation of logic in such a vein. However, our relativisation of what logic means through analogy is in position to change our mind in two ways: about what both logic and football are used for. The present paper is nothing but an introductory attempt to do so, by means of a comparative analysis of what logic and sports have fundamentally in common: being a game, without which these activities cannot make sense in a community of players.

\section{References}

[1] Belnap. N. “A Useful Four-Valued Logic", in Dunn M. (ed.), Modern Uses of MultipleValued Logic, Reidel, Boston: 8-37.

[2] Dummett, M. (1959). "Truth". Proceedings of the Aristotelian Society, Vol. 59: 141-62.

[3] Hintikka, J. (1982). "Game-theoretical semantics: insights and prospects", Notre Dame Journal of Formal Logic, Vol. 23: 219-41.

[4] Lorenzen, P. \& Lorenz, K. (1978). Dialogische Logik. Darmstadt: Wissenschaftliche Buchgesellschaft.

[5] Moktefi, A. \& Schang, F. (2015). "On Rules and Refereeing in Football”, in The Road of Universal Logic, Studies in Universal Logic, Springer, 2015: 407-15.

[6] Quine, W.V.O. (1982). Methods of Logic. Harvard University Press. 
[7] Schang, F. (2010). "Beyond the Fregean Myth: The Value of Logical Values", in Stalmasczyk, P. (ed.), Philosophy of Language and Linguistics, Volume I: The Formal Turn. Frankfurt: Ontos Verlag: 245-60.

[8] Schang, F. (2013). "A One-Valued Logic for Non-One-Sidedness", International Journal of Jaina Studies, Vol. 9(1): 1-25.

[9] Schang, F. (2017). "A Four-Valued Logic of Strong Conditional”, submitted draft.

[10] Schang, F. \& Chiffi, D. (2017). "The Logical Burdens of Proof. Assertion and Hypothesis", Logic and Logical Philosophy, Vol. 26(2): 1-22.

[11] Schang, F. \& Costa-Leite, A. (2016). "Une Sémantique Générale des Croyances Justifiées", CLE-prints, Vol. 16(3): 1-24.

[12] Schang, F. \& Trafford (201X), J. "Is 'no' a force indicator? Yes, sooner or later", forthcoming in Logica Universalis.

[13] Searle \& Vanderveken (2005). "Speech Acts and Illocutionary Logic", in Logic, Thought and Action, Vanderveken, D. (ed.), Cambridge University Press: 119-132.

[14] Vidal, M. (2014). "The defective conditional in mathematics", Journal of Applied NonClassical Logics, Vol. 24: 169-79.

\section{Appendix: The Meaning of Logical Constants in a Football of Logic.}

For every sentence $\varphi\left(p, q, p \bullet q\right.$, etc.) of the form $\mathbf{A}(\varphi)=\left\langle\mathbf{a}_{1}(\varphi), \mathbf{a}_{2}(\varphi)\right\rangle$, the logical constants - $=\{\neg, \wedge, \vee, \rightarrow\}$ of $\mathbf{A} \mathbf{R}_{4}$ are defined by their truth- and falsity-conditions. In the left part of the definitions, the left and right items of ordered pairs $\langle x, y\rangle$ express the truth- and falsity-conditions of the corresponding compound sentence $p \bullet q$; respectively. In the right part, the football logos are twofold: players having the ball and attacking (going forward, from left to right) symbolize truth; players deprived from the ball and defending (going backward, from right to left) symbolize falsity. The top schemes stand for truth-conditions, and the bottom schemes for falsity-conditions.

\section{Negation}

$\mathbf{A}(\neg p)=\left\langle\mathbf{a}_{2}(p), \mathbf{a}_{1}(p)\right\rangle$

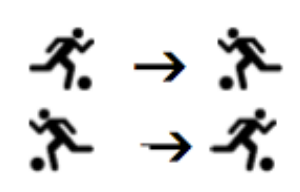

\section{Conjunction}

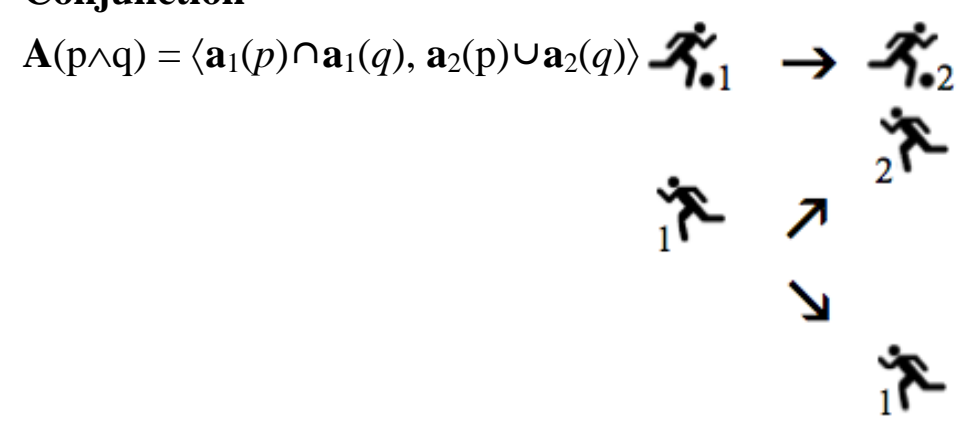




$$
\begin{aligned}
& \text { Disjunction } \\
& \begin{array}{lll}
\mathbf{A}(p \vee q)=\left\langle\mathbf{a}_{1}(p) \cup \mathbf{a}_{1}(q), \mathbf{a}_{2}(\mathrm{p}) \cap \mathbf{a}_{2}(q)\right\rangle \stackrel{\boldsymbol{\gamma}_{\bullet 1}}{\bullet} & \nearrow \\
& \searrow
\end{array} \\
& \mathcal{X}^{\circ} \\
& \underset{1}{i} \rightarrow \underset{2}{i} \\
& x_{.1} \rightarrow x_{02}
\end{aligned}
$$

